\title{
Wideband signal design for over-the-horizon radar in cochannel interference
}

\author{
Zhongtao Luo ${ }^{1 *}$, Kun Lu$^{2}$, Xuyuan Chen ${ }^{2}$ and Zishu He${ }^{1}$
}

\begin{abstract}
Ship detection in heavy sea clutter is a big challenge for over-the-horizon (OTH) radar. Wideband signal is helpful for improving range resolution and the signal-to-clutter ratio. In this paper, to support OTH radar employing wideband in cochannel interference, we propose environmental sensing-based waveform (ESBW) strategy, by considering transmit waveform design as an active approach and cognitive loop for the time-varying environment. In ESBW strategy, OTH radar monitors the environment in real time, estimates interference characteristics, designs transmit waveform adaptively, and employs traditional signal processing structure to detect targets in the presence of interference. ESBW optimization problem employs the criteria of maximizing the output signal-to-interference-plus-noise ratio (SINR) of matched filter and similarity constraint for reasonable range resolution and sidelobe levels. The analytic solution to this constrained problem is developed, so that ESBW design algorithm's efficiency is guaranteed, with adjustable SINR and autocorrelation function. A simulated scenario with strong interference and colored noise has been introduced. Simulation results demonstrate that OTH radar with ESBW strategy detects the target successfully in the background of cochannel interference.
\end{abstract}

Keywords: OTH radar; Waveform design; Interference suppression; Signal-to-interference-plus-noise ratio (SINR)

\section{Introduction}

Sky-wave over-the-horizon (OTH) radar makes use of the propagation through the ionosphere and is capable of detecting targets at long ranges from about 500 to $3,000 \mathrm{~km}$, accepted as effective wide-area surveillance sensors $[1,2]$. OTH radar operates in the high frequency (HF) band (5 30 MHz) where the external electromagnetic environment is space-time variant, surrounded by radio frequency interference (RFI) and atmospheric, cosmic, and man-made noise [3,4]. Sometimes, OTH radar has to operate in congested bands which are densely populated due to the use of radio frequencies [5].

For detecting slowly-moving vessels, OTH radar requires broader bandwidth than that in plane detection, since the Doppler shifts of ship echoes coincide within the spectrum range of sea clutter, which makes adequate signal-to-clutter ratio (SCR) essentially important for ship detection [6]. Broad bandwidth reduces the range resolution of sea scatter cell size so as to improve the SCR

*Correspondence: loztsky@163.com

1 University of Electronic Science and Technology of China, Xiyuan Ave, West

$\mathrm{Hi}$-Tech Zone, 611731 Chengdu, China

Full list of author information is available at the end of the article
[7]. However, when OTH radar employs wideband signal, the broad-occupying bandwidth increases the possibility of encountering cochannel interference.

To avoid cochannel interference, the frequency management system (FMS) is necessary in OTH radar for providing information on channel occupancy and noise level to select operating frequency $[1,3,4]$. Saverino et al. [8] proposes a cognitive waveform technique of selecting waveform parameters, such as bandwidth, pulse length, etc., based on 'available clear channels' determined by the FMS. The best choice for OTH radar is to avoid cochannel interference, if possible. However, 'avoiding' may not work sometimes when there is no unoccupied channel with sufficient bandwidth at all. In this case, insisting on clear narrowband leads to reduced bandwidth and increased sea-clutter power. Alternatively, employment of occupied wideband does not worsen the sea-clutter. Meanwhile, it requires interference suppression or signal design.

Interference cancelation algorithms have been proposed for eliminating cochannel interference in signal processing stage. Fabrizio proposes adaptive beamforming techniques in spatial domain $[9,10]$. Suppressing interference in time domain and frequency domain fundamentally

\section{Springer}

(c) 2014 Luo et al. licensee Springer. This is an Open Access article distributed under the terms of the Creative Commons

Attribution License (http://creativecommons.org/licenses/by/4.0), which permits unrestricted use, distribution, and reproduction in any medium, provided the original work is properly credited. 
involves estimating parameters of interference and then suppressing, with iterative algorithm widely employed, like least-mean-square filters $[11,12]$ and orthogonal subspace projection filtering [13]. Guo evaluates the interference cancelation performance of various schemes based on the minimum variance distortionless response (MVDR) criterion, in both time domain and Doppler domain [14]. However, as Fabrizio points out, the performance of adaptive beamforming is highly dependent on spatial characteristics of interference and limited by the receive array. The mutual problem shared by iterative algorithms above is how to guarantee that the interference components are suppressed 'enough' while the information of targets is preserved. In Guo's schemes, there is one requirement that interference training should be performed with ocean/ground clutter and strong targetlike components excluded, which is a hard task in skywave OTH radar. Besides, for interference cancelation performed on received data, there is one potential risk that employing nonlinear algorithm too much in signal process may cause unexpected influences, e.g., degrading clutter visibility [9]. Also, algorithms designed for suppressing narrowband interference are unsuitable for broadband interference or colored noise with diffused energy.

Unlike passive cancelation, transmit waveform design is an active approach, capable of avoiding reserved bands occupied by interference inside the transmit band. One example is disjoint spectrum waveform (DSW), however, giving rise to high range sidelobes which cannot be suppressed by traditional spectral weighting. Thus, DSW design is necessary, to balance desired spectra with low sidelobes. Transmit and receive waveforms are designed separately in [15]. SCAN (stopband cyclic algorithm new) and radar-centric design are proposed in $[16,17]$. However, DSW design algorithms do not work in an adaptive way. Also, it is a problem to define stopband for DSW design in practical application, especially in the presence of colored noise with spreading spectrum.

Adaptivity is important for waveform design in OTH radar due to the time-varying environment. This makes environmental sensing essential, to design waveform in a cognitive way. Cognitive radar is introduced for the first time by Simon Haykin in [18]. In our previous work [19], cognitive OTH radar (COTHR) is proposed for better frequency management and multiple-task performance. Herein, we consider employing cognition in wideband signal design in the background of cochannel interference.

Researchers have developed waveform design methods in colored noise with known statistical properties, under certain characteristics requirements, mainly involving constant modulus and autocorrelation function (ACF)
[20-22]. Numeric techniques and iterative algorithms are necessarily employed for this non-linear constrained waveform optimization. Motivated by Sussman's work [23], similarity constraint forces the sought-after waveform to be close in a certain sense to a desired waveform for reasonable ACF [22]. Similarity constraint in the infinity norm is employed in phased code design $[24,25]$. Similarity constraint in Euclidean norm is employed for robust receive beamforming [26] and waveform design based on whitening filter [27]. In this paper, we employ similarity constraint in Euclidean norm in waveform design based on matched filter, to control the output envelope effectively. The optimal waveform for maximizing output SINR under similarity constraint is deduced and given in closed form.

The remainder of this paper is organized as follows. In Section 2, we present the scheme of OTH radar system employing ESBW strategy, essentially summarized as environment monitoring, characteristics estimating, waveform design, and conventional operation. In Section 3, the waveform design algorithm for optimizing SINR under similarity constraint is developed, and the analytic and optimal solution is obtained as ESBW. In Section 4, a scenario with strong interference and colored noise is simulated as the external environment of OTH radar, by which we investigate the performances of ESBW strategy, and further the evaluation of user parameters. Finally, main conclusions are drawn in Section 5.

\section{OTH radar scheme with ESBW strategy}

Cognitive radar constitutes a dynamic closed cycle and emphasizes interaction between illuminating environment (targets, clutter, external interference, and background noise) and radar system (the transmitter, receiver, and signal processor) [18]. The transmitter adjusts the illumination continuously, according to what the receiver learns about the environment. Inspired by cognitive radar, our proposed environmental sensing-based waveform (ESBW) strategy works in a cognitive way, consisting of 'learning' and 'adjusting' by environmental sensing and adaptive waveform design, respectively. This leads us to the block diagram in Figure 1 which depicts the structures of OTH radar scheme in conventional way $[1,28]$ and another with ESBW strategy.

In conventional way, the frequency management system (FMS) monitors the ionosphere and background surrounding and selects waveform parameters, including operating frequency, bandwidth, pulse length, and repetition period. Then, the transmitter generates predetermined waveform, e.g. the widely used linear frequency modulated continuous waveform (LFMCW), modulates it to the operating frequency and emits high-frequency 


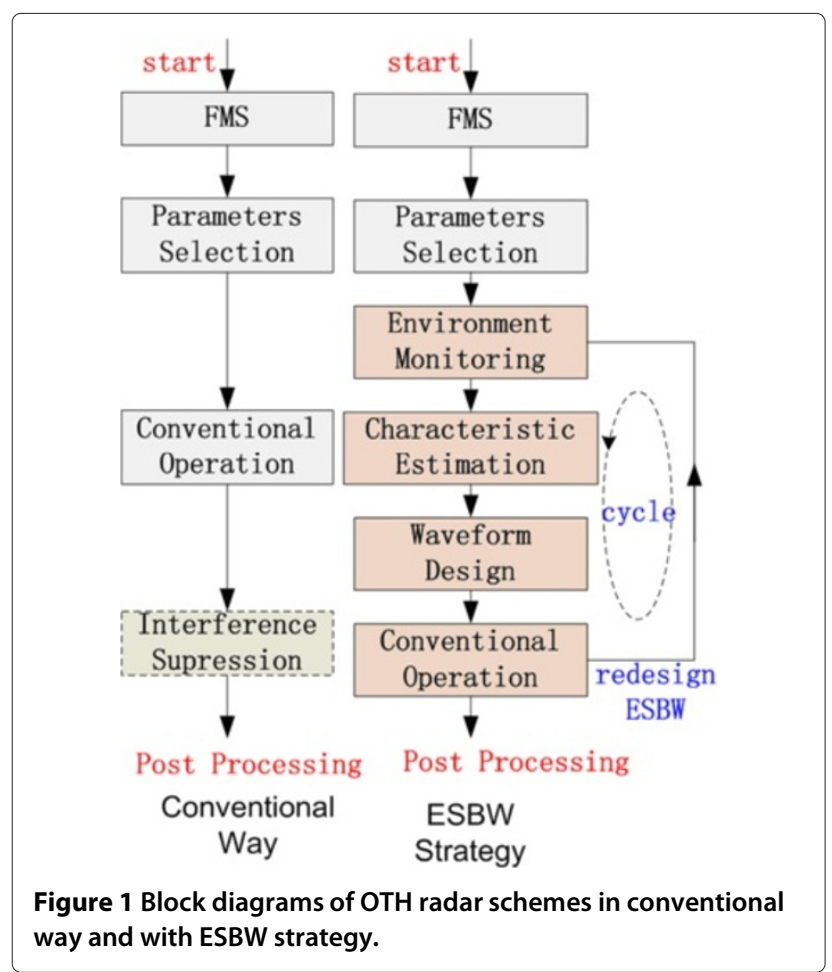

electromagnetic waves. Each antenna of receive array is configured to a digital receiver whose received data is sent to the high-speed signal processor, including digital beamforming (DBF), matched filter (MF), and Doppler processing basically. Ionospheric decontamination and clutter cancelation are employed for detecting slowlymoving vessels in a long coherent integration time (CIT). Interference cancelation may be involved if necessary, followed by target detection and tracking algorithms in the post-processing stage.

As for ESBW strategy, the radar scheme keeps the conventional structure with two exceptions. Firstly, after the FMS selects waveform parameters, ESBW strategy monitors the environment in real time by the receive array, and the designs transmit waveform adaptively based on the sensing results, instead of generating predetermined waveform. Secondly, there is no need of interference cancelation in signal processing (transient interference suppression is not included in ESBW strategy). Depending upon the environmental change, ESBW strategy operates in a loop consisting of environment monitoring, characteristic estimating, waveform design, and conventional operation, as described in the following.

\subsection{Environment monitoring}

Environment monitoring collects the environment data for characteristic analysis. To avoid the overwhelming sea clutter, environment monitoring is accomplished under radio silence when the transmitter is cut off.

Assume that the FMS suggests operating frequency $f_{c}$ and the mission requires bandwidth $B$. As the transmitter is cut off, the receive array, a uniform linear array (ULA) with $d$ spacing between adjacent antennas, monitors the electromagnetic environment at center frequency $f_{c}$ with bandwidth $B$ and modulates the received signal to the base band. Denote the sampling frequency and monitoring time as $f_{s}$ and $T_{s}$ respectively, and then the temporal sampling number is $N_{s}=f_{s} \times T_{s}$. Arrange the data sampled by the array at the $n_{s}$ th sample time, for $n_{s}=1,2, \ldots, N_{s}$, in a vector

$$
\widetilde{r}_{i, n_{s}}=\left[\widetilde{r}_{i}, 1 n_{s}, \widetilde{r}_{i, 2 n_{s}}, \ldots, \widetilde{r}_{i}, K n_{s}\right]
$$

where $K$ denotes the number of receive antennas. Then, the data sampled in the whole monitoring time is given in a matrix

$$
\widetilde{\boldsymbol{r}}_{\boldsymbol{i}}=\left[\widetilde{\boldsymbol{r}}_{\boldsymbol{i}, 1}^{T}, \widetilde{\boldsymbol{r}}_{\boldsymbol{i}, 2}^{T}, \ldots, \widetilde{\boldsymbol{r}}_{\boldsymbol{i}, N_{s}}^{T}\right]
$$

of dimension $K \times N_{s}$, where $(\cdot)^{T}$ denotes the transpose.

Herein, there is one principle for evaluating the monitoring time $-T_{s}$ should be as small as possible to reduce its negative effect on the illumination time, under the condition of effectively estimating the statistic characteristic of interference and noise. Otherwise, when $T_{s}$ exceeds a reasonable value, it would not bring significant gain on interference suppression but only reduce the illumination time of OTH radar.

Besides, there may be strong transient interference, such as lightning, in OTH radar environment. Transient interference detection algorithms $[29,30]$ could be employed to examine the data matrix $\widetilde{\boldsymbol{r}}_{\boldsymbol{i}}$. If it is confirmed that $\widetilde{\boldsymbol{r}}_{\boldsymbol{i}}$ contains transient interference, radar operators can remove the unwanted samples or simply monitor again.

\subsection{Characteristic estimation}

Characteristic estimation analyzes the environment data and provides information for waveform design. Since waveform design algorithm proposed in this paper is based on the temporal covariance of environment interference and noise, the data matrix $\widetilde{\boldsymbol{r}}_{i}$ is applied for covariance matrix estimate $\widehat{\boldsymbol{R}}_{\boldsymbol{i}}$. In spatial beamforming of $\widetilde{\boldsymbol{r}}_{\boldsymbol{i}}$, vector $\widetilde{\boldsymbol{w}}$ is employed, which equals to the receive beamforming vector $w$

$$
\widetilde{\boldsymbol{w}}=\boldsymbol{w}=\left[e^{-j 2 \pi f_{c} / c \cdot d \sin \varphi \cdot 0}, \ldots, e^{-j 2 \pi f_{c} / c \cdot d \sin \varphi \cdot(K-1)}\right]
$$


where $c$ denotes the speed of light, and $\varphi$ denotes the direction of receive beamforming. The output of spatial beamforming is

$$
\widetilde{\boldsymbol{I}}=\widetilde{\boldsymbol{w}} \cdot \widetilde{\boldsymbol{r}}_{\boldsymbol{i}},
$$

an $N_{s}$-dimensional vector.

Suppose the sampling number of seeking discrete waveform is $M=T \times f_{s}$, where $T$ denotes the pulse length. For $N_{s} \geq M$, covariance function is estimated by

$$
\widehat{R}(m)=\frac{1}{N_{s}} \sum_{l=1}^{N_{s}-m} \widetilde{I}(l+m) \widetilde{I}^{*}(l), 0 \leq m \leq M-1,
$$

where $\widetilde{I}(l)$ denotes the $l$ th element of $\widetilde{\boldsymbol{I}}$, and $(\cdot)^{*}$ denotes the conjugation. For $N_{s}<M$, covariance function is estimated by

$$
\widehat{R}(m)=\left\{\begin{array}{cc}
\frac{1}{N_{s}} \sum_{l=1}^{N_{s}-m} \widetilde{I}(l+m) \widetilde{I}^{*}(l), & 0 \leq m \leq N_{s}-1, \\
0, & N_{s} \leq m \leq M-1 .
\end{array}\right.
$$

Given the estimate $\widehat{R}(m)$, the covariance matrix estimate is obtained as a Toeplitz matrix

$$
\widehat{\boldsymbol{R}}_{\boldsymbol{i}}=\left[\begin{array}{cccc}
\widehat{R}(0) & \widehat{R}^{*}(1) & \cdots & \widehat{R}^{*}(M-1) \\
\widehat{R}(1) & \widehat{R}(0) & \cdots & \widehat{R}^{*}(M-2) \\
\vdots & \vdots & \ddots & \vdots \\
\widehat{R}(M-1) & \widehat{R}(M-2) & \cdots & \widehat{R}(0)
\end{array}\right]
$$

where $\widehat{\boldsymbol{R}}_{\boldsymbol{i}}=\widehat{\boldsymbol{R}}_{\boldsymbol{i}}^{H}$, and $(\cdot)^{H}$ denotes the transpose and conjugation operation.

\subsection{Waveform design}

ESBW strategy tends to suppress environmental interference by the transmit waveform. For the architecture of OTH radar with ESBW strategy, the figure of merit for a particular discrete time waveform is the SINR at the output of the envelope at the true target delay and Doppler shift [31]. Considering the SINR at the output of array signal processing (receive beanforming, MF, and Doppler processing), the optimization problem of waveform design can be formulated as follows:

$$
\min _{\boldsymbol{s}} \boldsymbol{s}_{\boldsymbol{i}} \boldsymbol{s}^{H}, \quad \text { s.t. } \quad \boldsymbol{s} \boldsymbol{s}^{H}=1
$$

where $s$ denotes an arbitrary discrete time waveform with norm 1 (see Appendix for details).

However, the so-obtained optimal solution to optimization problem (8) probably results in poor properties, e.g. poor range resolution and high sidelobe levels. Generally, radar waveform optimization should consider the characteristics of the solution besides the SINR gain. The proposed waveform design based on MF in this paper has one advantage which is: requirements on mainlobe width and sidelobe levels are equivalent to requirements on ACF. Otherwise, waveform design based on unmatched filter (including whitening filter) needs to consider crosscorrelation function (CCF) between the transmit waveform and corresponding filter response. Naturally, we think waveform design based on MF is more manageable than that based on unmatched filter, since the former refers to one factor while the latter refers to two.

Optimization problem, which considers problem (8) and ACF constraints jointly, becomes a non-linear constrained optimization and its analytic solution is unavailable. Herein, similarity constraint is employed for the constraints on ACF. By forcing the solution to be close in the Euclidean sense to some other waveform that possesses a desirable ACF, similarity constraint controls the waveform ACF indirectly [23]. In similarity constraint application, choose a waveform with desirable ACF, e.g., the linear frequency modulated (LFM) waveform, as the desired waveform $s_{0}$. Without loss of generality, it is assumed that $s_{0} s_{0}^{H}=1$. Then, the constrained optimization problem is given by

$$
\min _{\boldsymbol{s}} \boldsymbol{s}_{\boldsymbol{R}} \boldsymbol{s}^{H}, \quad \text { s.t. } \quad\|\boldsymbol{s}\|^{2}=1, \quad\left\|\boldsymbol{s}-\boldsymbol{s}_{0}\right\|^{2} \leq \varepsilon,
$$

where $\|\cdot\|$ denotes the Euclidean norm, and $\varepsilon$ is a user parameter which determines the degree of similarity between the solution and the desired waveform. The optimal and analytic solution to problem (9) is used to evaluate ESBW, denoted as $\boldsymbol{s}_{E}$. The detailed solution to problem (9) will be provided in Section 3.

\subsection{Conventional operation}

In conventional operation, $\mathrm{OTH}$ radar illuminates the area of interest by EBSW $\boldsymbol{s}_{E}$ and employs the conventional signal processing structure to detect targets.

Assume the data received by $K$ antennas in a CIT given in a matrix

$$
\widetilde{\boldsymbol{r}}\left(t_{n}\right)=\left[\begin{array}{cccc}
\widetilde{r}_{11} & \tilde{r}_{12} & \cdots & \widetilde{r}_{1 N} \\
\tilde{r}_{21} & \tilde{r}_{22} & \cdots & \tilde{r}_{2 N} \\
\vdots & \vdots & \ddots & \vdots \\
\tilde{r}_{K 1} & \widetilde{r}_{K 2} & \cdots & \tilde{r}_{K N}
\end{array}\right]
$$

where $t_{n}$ denotes the sample time, $N=P \times M$, and $P$ denotes the number of periods in a CIT.

In signal processing, vector $\boldsymbol{w}$ in (3) is employed for receive beamforming, waveform $\boldsymbol{s}_{E}$ for moving filter, and the fast Fourier transform (FFT) for Doppler processing. The output of delay-Doppler cell $\left(\tau^{\prime}, f_{d}^{\prime}\right)$ is given by

$$
\widetilde{y}\left(\tau^{\prime}, f_{d}^{\prime}\right)=\boldsymbol{w} \cdot \widetilde{\boldsymbol{r}}\left(t_{n}\right) \cdot \boldsymbol{s}_{\mathrm{Ft}, E}^{H}\left(t_{n}, \tau^{\prime}, f_{d}^{\prime}\right)
$$


where

$$
\begin{aligned}
& \boldsymbol{s}_{\mathrm{Ft}, E}\left(t_{n}, \tau^{\prime}, f_{d}^{\prime}\right) \\
& =\left[\boldsymbol{s}_{E}\left(t_{n}-\tau^{\prime}\right) e^{j 2 \pi f_{d}^{\prime} 0 T}, \ldots, \boldsymbol{s}_{E}\left(t_{n}-\tau^{\prime}\right) e^{j 2 \pi f_{d}^{\prime}(P-1) T}\right],
\end{aligned}
$$

and $\boldsymbol{s}_{E}\left(t_{n}-\tau^{\prime}\right)$ denotes the result of waveform $\boldsymbol{s}_{E}$ undergoing lag $\tau^{\prime}$.

In post processing, target detection algorithm (e.g., constant false alarm rate detection algorithm) is employed to examine the detection signal-to-noise ratio (SNR) of delay-Doppler $\left(\tau^{\prime}, f_{d}^{\prime}\right)$ cells, followed by data process algorithms, such as target tracking and coordinate registration [28].

\section{Waveform design algorithm}

The constrained optimization problem (9) of ESBW design is rewritten here as

$$
\min _{\boldsymbol{s}} \boldsymbol{s}_{\boldsymbol{i}} \boldsymbol{s}^{H}, \quad \text { s.t. } \quad\|\boldsymbol{s}\|^{2}=1, \quad\left\|\boldsymbol{s}-\boldsymbol{s}_{0}\right\|^{2} \leq \varepsilon .
$$

Compute the eigendecomposition of $\widehat{\boldsymbol{R}}_{\boldsymbol{i}}$ as

$$
\widehat{\boldsymbol{R}}_{\boldsymbol{i}}=\boldsymbol{U} \boldsymbol{\Lambda} \boldsymbol{U}^{H}
$$

where the columns of $\boldsymbol{U}$ contain the eigenvectors of $\widehat{\boldsymbol{R}}_{\boldsymbol{i}}$. Diagonal matrix $\boldsymbol{\Lambda}=\operatorname{diag}\left\{\Lambda_{1}, \Lambda_{2}, \cdots, \Lambda_{M}\right\}$ lists the eigenvalues of $\widehat{\boldsymbol{R}}_{i}, \Lambda_{1} \geq \Lambda_{2} \geq \cdots \geq \Lambda_{M}>0$. The $m$ th column vector of $\boldsymbol{U}$ corresponds to the eigenvalue $\Lambda_{m}$. It is well-known that the minimization of $\boldsymbol{s} \widehat{\boldsymbol{R}}_{i} \boldsymbol{s}^{H}$ is obtained at $\boldsymbol{s}=\boldsymbol{u}_{M}^{T}$, for

$$
\boldsymbol{s} \widehat{\boldsymbol{R}}_{i} \boldsymbol{s}^{H} \geq \boldsymbol{u}_{M}^{T} \widehat{\boldsymbol{R}}_{i} \boldsymbol{u}_{M}^{*}=\Lambda_{M} .
$$

Though $\boldsymbol{s}=\boldsymbol{u}_{M}^{T}$ is the optimal solution to problem (8), generally $\boldsymbol{u}_{M}^{T}$ bears wide mainlobe and low peak to sidelobe ratio for a radar waveform. Alternatively speaking, $\boldsymbol{s}=\boldsymbol{u}_{M}^{T}$ dissatisfies the constraint $\left\|\boldsymbol{s}-\boldsymbol{s}_{0}\right\|^{2} \leq \varepsilon$ in (13) for a reasonable $\varepsilon$. In the following, we will provide the solution to problem (13) for $\left\|\boldsymbol{u}_{M}^{\mathrm{T}}-\boldsymbol{s}_{0}\right\|^{2}>\varepsilon$, which resembles a sort of doubly constrained robust Capon beamformer in [26].

By unit energy assumption $\|\boldsymbol{s}\|^{2}=\left\|\boldsymbol{s}_{\mathbf{0}}\right\|^{2}=1$, problem

(13) can be rewritten as

$$
\min _{\boldsymbol{s}} \widehat{s}_{\boldsymbol{R}_{i}} \boldsymbol{s}^{H}, \quad \text { s.t. } \quad \boldsymbol{s} \boldsymbol{s}^{H}=1, \boldsymbol{s s}_{0}^{H}+\boldsymbol{s}_{0} \boldsymbol{s}^{H} \geq 2-\varepsilon .
$$

Lagrange multiplier is employed to solve this problem. Consider the function

$$
f_{1}(\boldsymbol{s}, \lambda, \mu)=\boldsymbol{s} \widehat{\boldsymbol{R}}_{i} \boldsymbol{s}^{H}+\lambda\left(\boldsymbol{s} \boldsymbol{s}^{H}-1\right)+\mu\left(2-\varepsilon-\boldsymbol{s} \boldsymbol{s}_{0}^{H}-\boldsymbol{s}_{0} \boldsymbol{s}^{H}\right)
$$

where $\lambda$ and $\mu$ are real-valued Lagrange multipliers, $\mu>$ 0 , yielding $f_{1}(\boldsymbol{s}, \lambda, \mu) \leq \boldsymbol{s} \widehat{\boldsymbol{R}}_{i} \boldsymbol{s}^{H}$. Besides, $\lambda$ satisfies

$$
\widehat{\boldsymbol{R}}_{\boldsymbol{i}}+\lambda \boldsymbol{E}>0
$$

where $\boldsymbol{E}$ denotes an $M$-rank identity matrix, so that the cost function can be minimized with respect to $s$. In equation (18), it implies that $\lambda$ is greater than the opposition of the minimum eigenvalue of $\widehat{\boldsymbol{R}}_{\boldsymbol{i}}$. The cost function (17) can be written as

$$
\begin{aligned}
f_{1}(\boldsymbol{s}, \lambda, \mu)= & {\left[\boldsymbol{s}-\mu \boldsymbol{s}_{0}\left(\widehat{\boldsymbol{R}}_{\boldsymbol{i}}+\lambda \boldsymbol{E}\right)^{-1}\right]\left(\widehat{\boldsymbol{R}}_{\boldsymbol{i}}+\lambda \boldsymbol{E}\right) } \\
& \cdot\left[\boldsymbol{s}-\mu \boldsymbol{s}_{0}\left(\widehat{\boldsymbol{R}}_{\boldsymbol{i}}+\lambda \boldsymbol{E}\right)^{-1}\right]^{H} \\
& -\mu^{2} \boldsymbol{s}_{0}\left(\widehat{\boldsymbol{R}}_{\boldsymbol{i}}+\lambda \boldsymbol{E}\right)^{-1} \boldsymbol{s}_{0}^{H}-\lambda+\mu(2-\varepsilon) .
\end{aligned}
$$

Since $\left(\widehat{\boldsymbol{R}}_{\boldsymbol{i}}+\lambda \boldsymbol{E}\right)$ is opposite definite, given $\lambda$ and $\mu$, the minimization of (19) is achieved by

$$
\boldsymbol{s}_{\lambda \mu}=\mu \boldsymbol{s}_{0}\left(\widehat{\boldsymbol{R}}_{\boldsymbol{i}}+\lambda \boldsymbol{E}\right)^{-1} .
$$

If the minimizers of $f_{1}(s, \lambda, \mu)$ are proper and the corresponding $\boldsymbol{s}_{\lambda \mu}$ satisfies the constraint in (13), we say that the optimal solution to (13) is found. The cost function (19) is updated into

$$
f_{2}\left(\boldsymbol{s}_{\lambda \mu}, \lambda, \mu\right)=-\mu^{2} \boldsymbol{s}_{0}\left(\widehat{\boldsymbol{R}}_{\boldsymbol{i}}+\lambda \boldsymbol{E}\right)^{-1} \boldsymbol{s}_{0}^{H}-\lambda+\mu(2-\varepsilon) .
$$

For the Hessian of $f_{2}\left(\boldsymbol{s}_{\lambda \mu}, \lambda, \mu\right)$ with respect to $(\lambda, \mu)$ is negative definite, $f_{2}\left(\boldsymbol{s}_{\lambda \mu}, \lambda, \mu\right)$ has a unique maximum which is the minimum of $f_{1}(\boldsymbol{s}, \lambda, \mu)$ obtained by $\boldsymbol{s}=\boldsymbol{s}_{\lambda \mu}$. Now, we need to find the value of $(\widehat{\lambda}, \widehat{\mu})$ which maximizes $f_{2}\left(\boldsymbol{s}_{\lambda \mu}, \lambda, \mu\right)$. By letting the differential of $f_{2}\left(\boldsymbol{s}_{\lambda \mu}, \lambda, \mu\right)$ with respect to $\mu$ equal to 0 , we arrive at

$$
\mu=\frac{2-\varepsilon}{2 \boldsymbol{s}_{0}\left(\widehat{\boldsymbol{R}}_{\boldsymbol{i}}+\lambda \boldsymbol{E}\right)^{-1} \boldsymbol{s}_{0}^{H}} .
$$

Plugging (22) in (21) yields

$$
f_{3}(\lambda)=-\lambda+\frac{(2-\varepsilon)^{2}}{4 \boldsymbol{s}_{0}\left(\widehat{\boldsymbol{R}}_{\boldsymbol{i}}+\lambda \boldsymbol{E}\right)^{-1} \boldsymbol{s}_{0}^{H}} .
$$

The differential of $f_{3}(\lambda)$ with respect to $\lambda$ is derived as

$$
\frac{\partial f_{3}(\lambda)}{\partial \lambda}=\left(1-\frac{\varepsilon}{2}\right)^{2} \frac{\boldsymbol{s}_{0}\left(\widehat{\boldsymbol{R}}_{\boldsymbol{i}}+\lambda \boldsymbol{E}\right)^{-2} \boldsymbol{s}_{0}^{H}}{\left[\boldsymbol{s}_{0}\left(\widehat{\boldsymbol{R}}_{\boldsymbol{i}}+\lambda \boldsymbol{E}\right)^{-1} \boldsymbol{s}_{0}^{H}\right]^{2}}-1 \equiv g(\lambda) .
$$

Now, we can solve the function $g(\lambda)=0$ for $\widehat{\lambda}$. Note that

$$
\begin{aligned}
\frac{\partial g(\lambda)}{\partial \lambda}= & \left(1-\frac{\varepsilon}{2}\right)^{2} \cdot\left\{\frac{\left[\boldsymbol{s}_{0}\left(\widehat{\boldsymbol{R}}_{\boldsymbol{i}}+\lambda \boldsymbol{E}\right)^{-2} \boldsymbol{s}_{0}^{H}\right]^{2}}{\left[\boldsymbol{s}_{0}\left(\widehat{\boldsymbol{R}}_{\boldsymbol{i}}+\lambda \boldsymbol{E}\right)^{-1} \boldsymbol{s}_{0}^{H}\right]^{3}}\right. \\
& \left.-\frac{\left[\boldsymbol{s}_{0}\left(\widehat{\boldsymbol{R}}_{\boldsymbol{i}}+\lambda \boldsymbol{E}\right)^{-3} \boldsymbol{s}_{0}^{H}\right]}{\left[\boldsymbol{s}_{0}\left(\widehat{\boldsymbol{R}}_{\boldsymbol{i}}+\lambda \boldsymbol{E}\right)^{-1} \boldsymbol{s}_{0}^{H}\right]^{2}}\right\}
\end{aligned}
$$


By using Cauchy-Schwarz inequality, we have

$$
\frac{\partial g(\lambda)}{\partial \lambda}<0
$$

Thus, $g(\lambda)$ is monotonically decreasing with respect to $\lambda$. Obliviously, as $\lambda \rightarrow \infty, g(\lambda) \rightarrow(1-\varepsilon / 2)^{2}-1<0$, and $\lambda \rightarrow-\Lambda_{M}^{+}, g(\lambda) \rightarrow(1-\varepsilon / 2)^{2} /\left|\boldsymbol{u}_{M}^{T} s_{0}^{H}\right|-1$. Moreover, since $\boldsymbol{s}=\boldsymbol{u}_{M}^{T}$ dissatisfies $\boldsymbol{s s}_{0}^{H}+\boldsymbol{s}_{0} \boldsymbol{s}^{H} \geq 2-\varepsilon$, we have $\Re\left(\boldsymbol{u}_{M}^{T} \boldsymbol{s}_{0}^{H}\right)<1-\varepsilon / 2$, where $\Re(\cdot)$ denotes the real part of a complex. Considering the spherical uncertainty set, the maximum of $\Re\left(\boldsymbol{u}_{M}^{T} s_{0}^{H}\right)$ satisfies the equality, so that $\left[\Re\left(\boldsymbol{u}_{M}^{T} s_{0}^{H}\right)\right]^{2} \leq\left|\boldsymbol{u}_{M}^{\mathrm{T}} \boldsymbol{s}_{0}^{H}\right|^{2}<(1-\varepsilon / 2)^{2}$, leading to $g\left(-\Lambda_{M}^{+}\right)>0$. Hence, there is a unique solution for the function $g(\lambda)=0$ given as

$$
\left(1-\frac{\varepsilon}{2}\right)^{2} \frac{\sum_{m^{\prime}=1}^{M}\left|z_{m^{\prime}}\right|^{2} /\left(\Lambda_{m^{\prime}}+\lambda\right)^{2}}{\left[\sum_{m^{\prime}=1}^{M}\left|z_{m^{\prime}}\right|^{2} /\left(\Lambda_{m^{\prime}}+\lambda\right)\right]^{2}}-1=0,
$$

where $z_{m^{\prime}}$ denotes the $m^{\prime}$ th element of vector $z_{0}$, for

$$
z_{0}=\boldsymbol{U} \boldsymbol{s}_{0}^{H} .
$$

Equation (27) can be solved for $\widehat{\lambda}$ efficiently via a numeric technique, like Newton's method with the differential function $\partial g(\lambda) / \partial \lambda$ in (25). Given $\widehat{\lambda}$, substitute (22) into (20), and the optimal solution is obtained as

$$
\boldsymbol{s}_{E}=\left(1-\frac{\varepsilon}{2}\right) \frac{\boldsymbol{s}_{0}\left(\widehat{\boldsymbol{R}}_{\boldsymbol{i}}+\widehat{\lambda} \boldsymbol{E}\right)^{-1}}{\boldsymbol{s}_{0}\left(\widehat{\boldsymbol{R}}_{\boldsymbol{i}}+\widehat{\lambda} \boldsymbol{E}\right)^{-1} \boldsymbol{s}_{0}^{H}},
$$

which satisfies the similarity constraint

$$
\boldsymbol{s}_{E} \boldsymbol{s}_{0}^{H}=1-\frac{\varepsilon}{2} .
$$

Hereby $s_{E}$ is a proper solution, for it belongs to the boundary of similarity constraint. The corresponding SINR is given by

$\operatorname{SINR}=\frac{1}{\boldsymbol{s}_{E} \widehat{\boldsymbol{R}}_{i} \boldsymbol{s}_{E}^{H}}=\frac{\left[\sum_{m=1}^{M}\left|z_{m}\right|^{2} /\left(\Lambda_{m}+\widehat{\lambda}\right)\right]^{2}}{(1-\varepsilon / 2)^{2} \cdot \sum_{m=1}^{M}\left|z_{m}\right|^{2} \Lambda_{m} /\left(\Lambda_{m}+\widehat{\lambda}\right)^{2}}$.

Finally, the ESBW design algorithm is summarized as the following steps:

(1) Compute the eigendecomposition of $\widehat{\boldsymbol{R}}_{\boldsymbol{i}}$. If the eigenvector $\boldsymbol{u}_{M}$ corresponding to the minimum eigenvalue satisfies the similarity constraint, evaluate ESBW as $\boldsymbol{s}_{E}=\boldsymbol{u}_{M}^{T}$. Otherwise, continue.

(2) Solve the function $g(\lambda)=0$ in (27) for $\widehat{\lambda}$. Numeric technique could be involved, e.g. Newton's method with the differential function $\partial g(\lambda) / \partial \lambda$ given in (25). The initial value for iteration could be chosen as little greater than $-\lambda_{M}$. On the contrary, an improper great initial value may lead to invalid value less than the lower bound of $\lambda$.

(3) ESBW is achieved by using $\widehat{\lambda}$ in (29).

\section{Simulations and analysis}

In this section, we illustrate the OTH radar scheme with ESBW strategy and analyze the performance of interference suppression. Firstly, a scenario consisting of OTH radar, strong interference, and colored noise is simulated. Then, EBSW strategy is illustrated, step by step. The ACF, power spectrum, and detection SNR of ESBW are investigated, comparing to LFMCW. Finally, user parameters $T_{s}$ and $\varepsilon$ are discussed under joint consideration of detection SNR and the ACF.

\subsection{Scenario simulation}

Three types of interference are simulated in the scenario. Interference 1 is an amplitude modulated signal

$$
i_{1}(t)=A_{1}(t) \cdot \exp \left(j 2 \pi f_{1} t+j \phi_{1}\right),
$$

where $A_{1}(t)=\cos (2 \pi \beta t)$ denotes a cosine envelope, $\beta$ denotes the modulating frequency, $f_{1}$ denotes the difference between the center frequency of interference 1 and operating frequency $f_{c}$, and $\phi_{1}$ denotes random initial phase uniformly distributed in $(0,2 \pi)$. Interference 1 is a point interference with an incidence angle $\theta_{1}$ with steering vector $\boldsymbol{b}_{1}=\boldsymbol{a}\left(\theta_{1}\right)$, where

$$
\boldsymbol{a}(\vartheta)=\left[e^{j 2 \pi f_{c} / c \cdot d \sin \vartheta \cdot 0}, \cdots, e^{j 2 \pi f_{c} / c \cdot d \sin \vartheta \cdot(K-1)}\right]
$$

denotes the receiving steering vector for incident angle $\vartheta$. Interference 2 is a phase code signal

$i_{2}(t)=\sum_{n_{2}=1} A_{2}\left(n_{2}\right) \cdot \operatorname{rect}\left[\left(t-n_{2} T_{2}\right) / T_{2}\right] \cdot \exp \left(j 2 \pi f_{2} t+j \phi_{2}\right)$,

where $\operatorname{rect}(\cdot)$ denotes a rectangular window, $\operatorname{rect}(t)=1$ for $t \in(0,1)$ and otherwise $\operatorname{rect}(t)=0$. And $T_{2}$ denotes the length of each code unit, $A_{2}\left(n_{2}\right)=1$ or -1 denotes two-phase code, $f_{2}$ denotes the difference between the center frequency of interference 2 and $f_{c}$, and $\phi_{2}$ denotes random initial phase uniformly distributed in $(0,2 \pi)$. Interference 2 is a point interference with an incidence angle $\theta_{2}$ with steering vector $\boldsymbol{b}_{2}=\boldsymbol{a}\left(\theta_{2}\right)$.

Interference 3 is an autoregressive process [21,22,27], generated by filtering circularly symmetric complexvalued white Gaussian noise with the filter

$$
H(z)=\frac{1}{\left(1-1.5 z^{-1}+0.7 z^{-2}\right)^{4}},
$$

where $z^{-1}$ denotes the unit delay operator. As colored noise, $i_{3}(t)$ is assumed to extend in wide-scale area. Its receiving steering vector $\boldsymbol{b}_{3}$ is a $K$-dimensional vector 
whose elements' phases are identically independently distributed (i.i.d.), uniformly in $(0,2 \pi)$.

Hereby, the array received signal model is given by

$$
\boldsymbol{r}_{\boldsymbol{i}}=\sum_{q=1}^{3} \alpha_{q} \boldsymbol{b}_{q}^{T} \boldsymbol{i}_{q}+\boldsymbol{n},
$$

where $\boldsymbol{n}$ denotes i.i.d. additive white Gaussian noise (AWGN) with zero mean and covariance 1 , and $\alpha_{q}$ denotes the complex amplitude of interference. The sum of three types of interference plus AWGN is called environmental noise in the following. Signal model of environmental noise in (36) applies in two steps: firstly in environment monitoring to simulate monitored samples and secondly in conventional operation to generate the environmental noise component of the received data.

In the following simulations, OTH radar parameters are set as $f_{c}=10 \mathrm{MHz}, B=40 \mathrm{kHz}, d=15 \mathrm{~m}, T=0.02 \mathrm{~s}$, and $K=200$. Interference parameters are set as $\beta=$ $1011 \mathrm{~Hz}, f_{1}=-10 \mathrm{kHz}, T_{2}=0.002 \mathrm{~s}, f_{2}=12 \mathrm{kHz}$, $\theta_{1}=9^{\circ}$, and $\theta_{2}=10^{\circ}$. We vary $\alpha_{q}$ to set the interferenceto-noise ratios (INRs) of interferences $i_{1}(t), i_{2}(t)$, and $i_{3}(t)$ equal to $0 \mathrm{~dB},-10 \mathrm{~dB}$, and $0 \mathrm{~dB}$ respectively in each antenna.

\subsection{Environmental sensing}

The environment monitoring time is set $T_{s}=0.04 \mathrm{~s}$. Monitoring data matrix $\widetilde{r}_{i}$ is simulated by (36).

To examine the performance of ESBW on suppressing interference in mainlobe, the interested direction angle is set $\varphi=\theta_{2}$. Then, beamforming vector $\widetilde{\boldsymbol{w}}=\boldsymbol{b}_{2}^{*}$ is employed to compute $\widetilde{\boldsymbol{I}}$. Covariance function estimate $\widehat{R}(m)$ is calculated by (5) for $T_{s}>T$, and then covariance matrix estimate $\widehat{\boldsymbol{R}}_{\boldsymbol{i}}$ is produced by (7). Normalized covariance function estimate $\widehat{R}(m)$ is depicted in Figure 2 . Most energy of $\widehat{R}(m)$ is centralized within $2 \mathrm{~ms}$, an interval much less than pulse length $T=20 \mathrm{~ms}$. Generally for improving transmitting energy and range unambiguity in ship detection, OTH radar employs continuous waveform with pulse length larger than $20 \mathrm{~ms}$. It is such a long time beyond which the interference covariance function approximates zero, approaching formula (53).

The power spectral density (PSD) estimate of environmental noise is shown in Figure 3. From Figure 3, we can see that the maximum of available clear band is about $10 \mathrm{kHz}$, much less than $B$. If employing clear band is insisted, then the sea scatter cell size will be increased significantly, as well as the sea-clutter power.

\subsection{ESBW design}

In waveform design algorithm, similarity parameter is set $\varepsilon=0.1$, and LFMCW with bandwidth $B=40 \mathrm{kHz}$ is chosen as the desired waveform $s_{0}=\exp [j \pi B t(t / T-$ 1)], $0<t<T$. ESBW is computed by the waveform

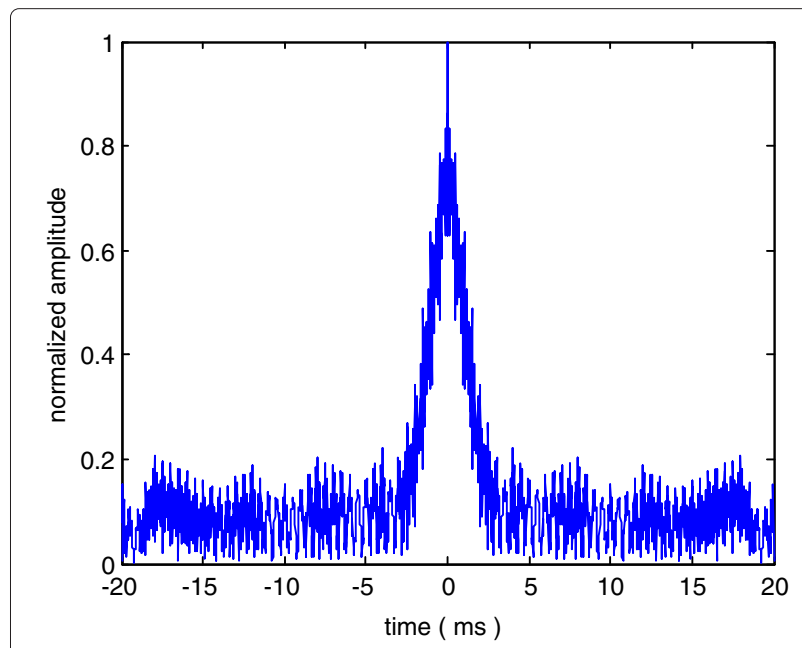

Figure 2 Covariance function estimate of environmental noise (after beamforming), $T_{s}=0.04$.

design algorithm given in Section 3, based on $\widehat{\boldsymbol{R}}_{\boldsymbol{i}}$ obtained in the previous subsection. Power spectra of ESBW and LFMCW are depicted in Figure 4, where the thin line and broad line denote power spectra of ESBW and LFMCW, respectively. It can be seen that ESBW concentrates its energy at the spectrum where the power of interference is relatively weak and decreases its energy at the peaks of environmental PSD. ESBW power spectrum has two nulls around $-10 \mathrm{kHz}$ corresponding to $i_{1}(t)$, one deep null at $12 \mathrm{kHz}$ to $i_{2}(t)$, and two shallow and wide notches around $0 \mathrm{kHz}$ to $i_{3}(t)$. It reveals that ESBW suppresses interference in frequency domain.

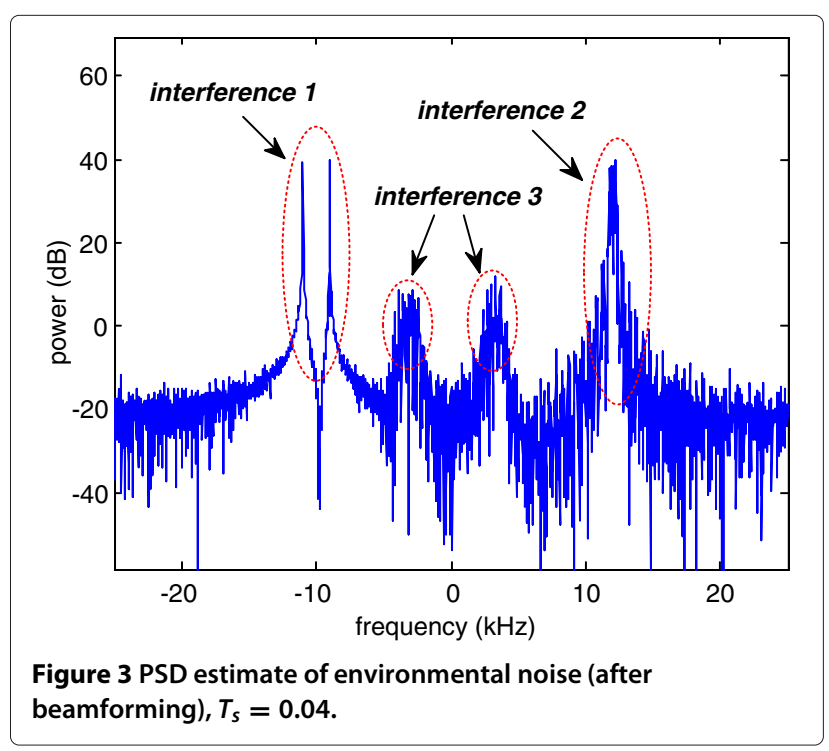




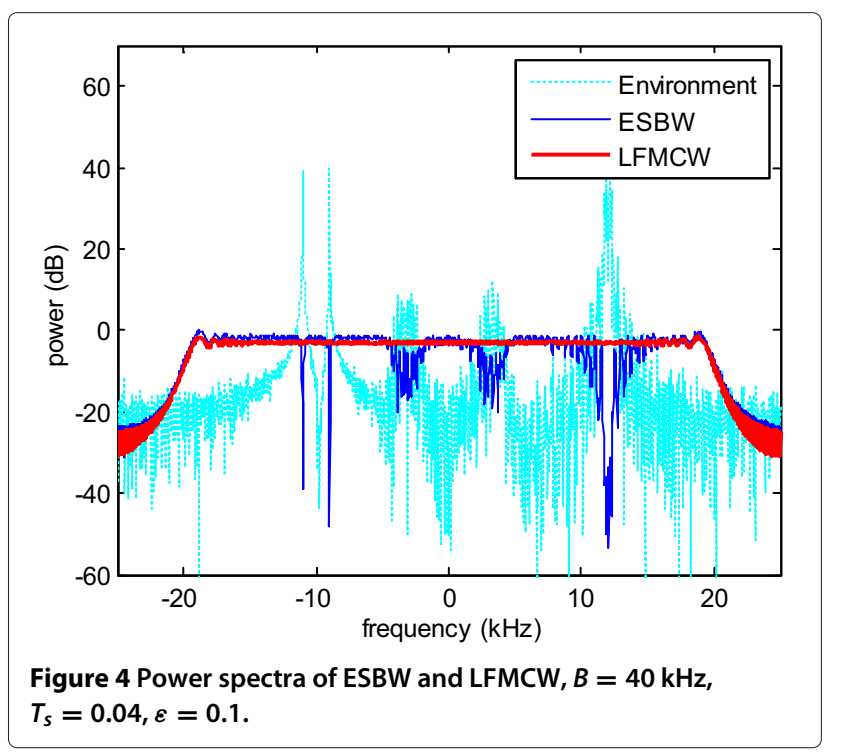

The ACFs of ESBW and LFMCW are compared in Figure 5. ESBW bears a reasonable ACF, with sidelobe levels about $-30 \sim-50 \mathrm{~dB}$, worse than LFMCW though. Figure 6 shows that the mainlobe widths of ESBW and LFMCW are close. It implies that ESBW design maintains the range resolution of the desired waveform basically. Hence, the designed ESBW is expected to keep the advantages of wideband LFMCW on broad bandwidth and high range resolution, and retain the sea-clutter power as LFMCW does.

\subsection{Signal processing results}

In this subsection, received data of target echo and environmental noise is simulated when OTH radar employs

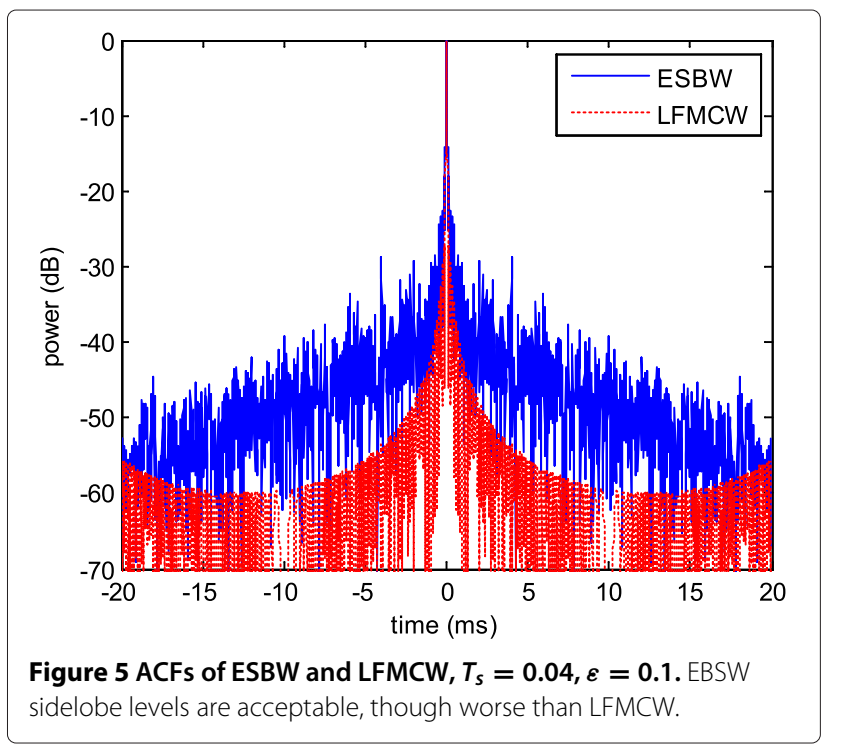

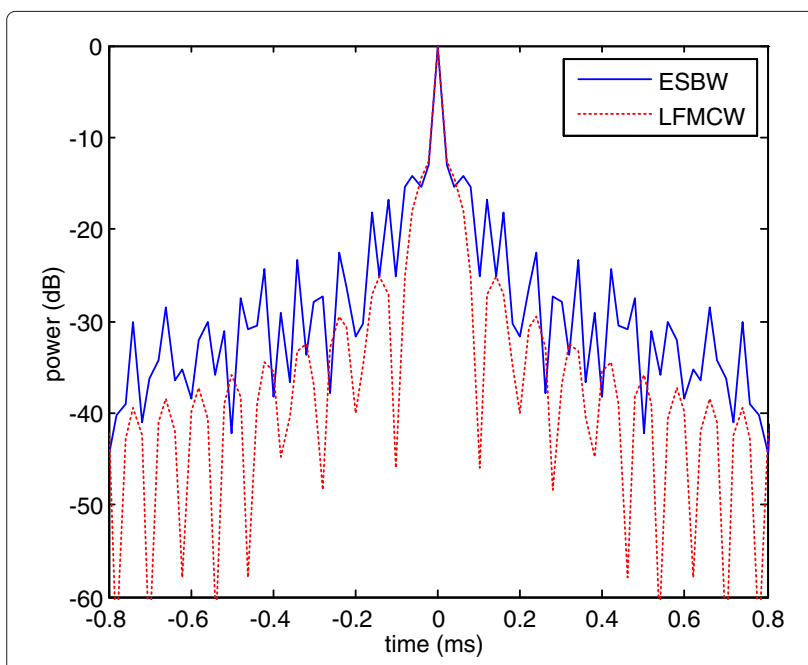

Figure 6 ESBW maintains the mainlobe width of LFMCW, $T_{s}=0.04, \varepsilon=0.1$.

ESBW to illuminate a target. Then, signal processing procedure is performed to show the range-velocity map and compute the detection SNR. For investigating ESBW's performance on interference suppression, same simulations are done for LFMCW to compare their results.

Assume that the echo is scattered by a target at slant range $R_{t}=1650 \mathrm{~km}$ and radial velocity $v_{t}=15 \mathrm{~m} / \mathrm{s}$, with incidence angle $\theta_{t}=10^{\circ}$. Then, the time delay, Doppler shift, and steering vector of target echo are $\tau=2 R_{t} / c$, $f_{d}=2 v_{t} f_{c} / c$ and $\boldsymbol{a}\left(\theta_{t}\right)$, respectively. The received signal model is given by

$$
\begin{aligned}
\tilde{\boldsymbol{r}}= & \boldsymbol{a}^{T}\left(\theta_{t}\right) \cdot \alpha_{t} \sum_{p=0}^{P-1} \operatorname{rect}[(t-\tau-p T) / T] \\
& \cdot s(t-\tau-p T) e^{j 2 \pi f_{d} t}+\widetilde{\boldsymbol{r}}_{\boldsymbol{i}}^{\prime}
\end{aligned}
$$

where $\widetilde{\boldsymbol{r}}_{\boldsymbol{i}}^{\prime}$ is environmental noise component generated by (35) newly with respect to the monitored data $\tilde{\boldsymbol{r}}_{\boldsymbol{i}}, s(t)$ denotes ESBW or LFMCW, and $\alpha_{t}$ denotes the complex amplitude of target echo. In the following, we set $P=200$ and vary $\alpha_{t}$ for SNR $=-55 \mathrm{~dB}$ in each antenna.

In signal processing, weighting vector $\boldsymbol{w}=\boldsymbol{b}_{2}^{*}$ is employed for receive beamforming, $\boldsymbol{s}_{0}$ and $\boldsymbol{s}_{E}$ for $\mathrm{MF}$, and FFT method for Doppler processing. The results in range-velocity map for LFMCW and ESBW are shown in Figures 7 and 8. In Figure 7 for LFMCW, two continuous peaks at velocity $\pm 165 \mathrm{~m} / \mathrm{s}$, which are produced by interference 1 due to the ambiguity Doppler frequency. The normalized power of target cell is $-5 \mathrm{~dB}$, so that the target is undetectable. Besides, there are many lower peaks distributed in mess all over the range-velocity map, owing to interferences 2 and 3. However, in Figure 8 for ESBW, 


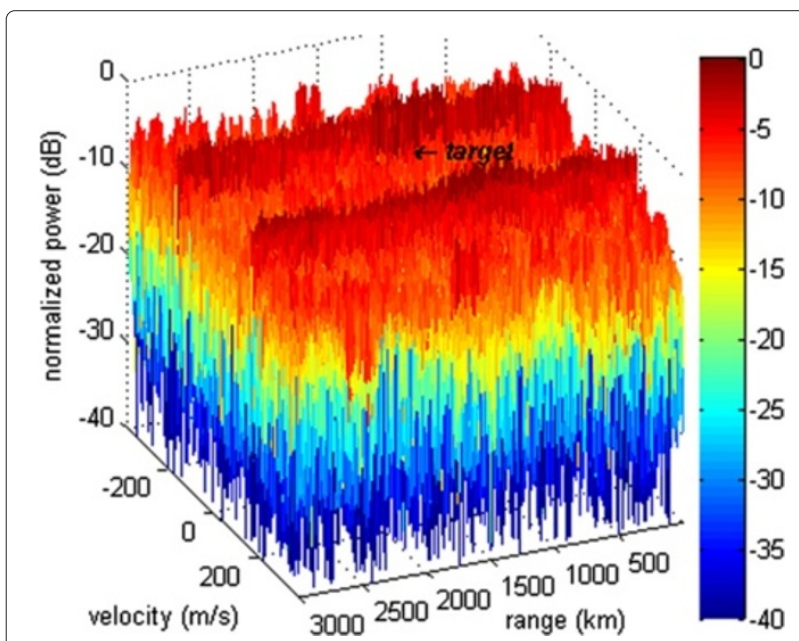

Figure 7 Range-velocity map employing LFMCW. A target is located at $R_{t}=1650 \mathrm{~km}$ with velocity $v_{t}=15 \mathrm{~m} / \mathrm{s}$, and $P=200$.

the peaks produced by interference are canceled, and the target is visible.

For detailed investigation, Figure 9 depicts the power along the range dimension at velocity $15 \mathrm{~m} / \mathrm{s}$, normalized with respect to the range cell where the target exists. The dashed line denotes LFMCW while the solid line denotes ESBW. We can see that the dashed line spreads over $-10 \sim 0 \mathrm{~dB}$ in all the range cells while the solid line gets a peak at the range of $1650 \mathrm{~km}$ with others mainly $-10 \mathrm{~dB}$ below. By employing cell-averaging constant false alarm rate (CA-CFAR) detector (cells of the same range or velocity are excluded), the calculated detection SNR of ESBW is $15.4 \mathrm{~dB}$, which implies that the target is detectable. Similarly, Figure 10 depicts the power along the velocity dimension at the range of $1650 \mathrm{~km}$,

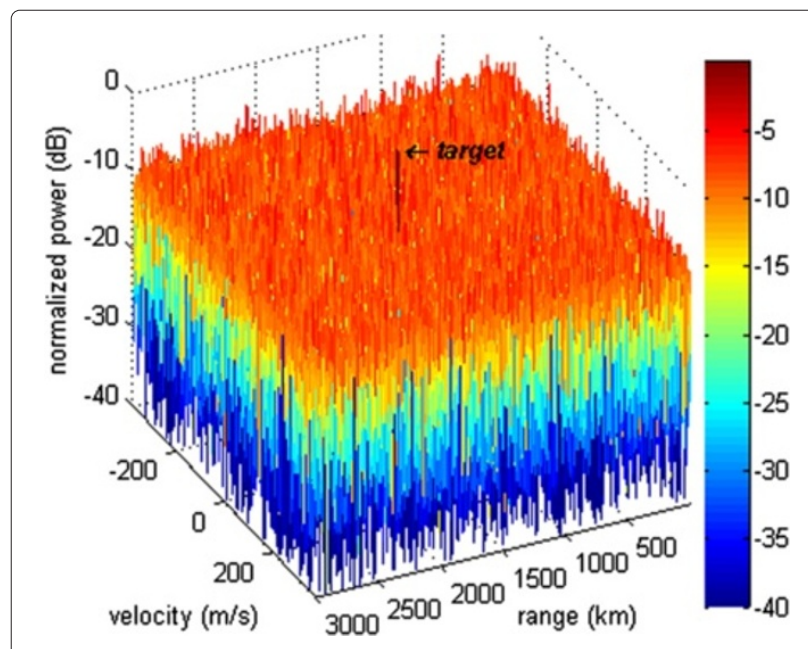

Figure 8 Range-velocity map employing ESBW. A target is located at $R_{t}=1650 \mathrm{~km}$ with velocity $v_{t}=15 \mathrm{~m} / \mathrm{s}$, and $P=200$.

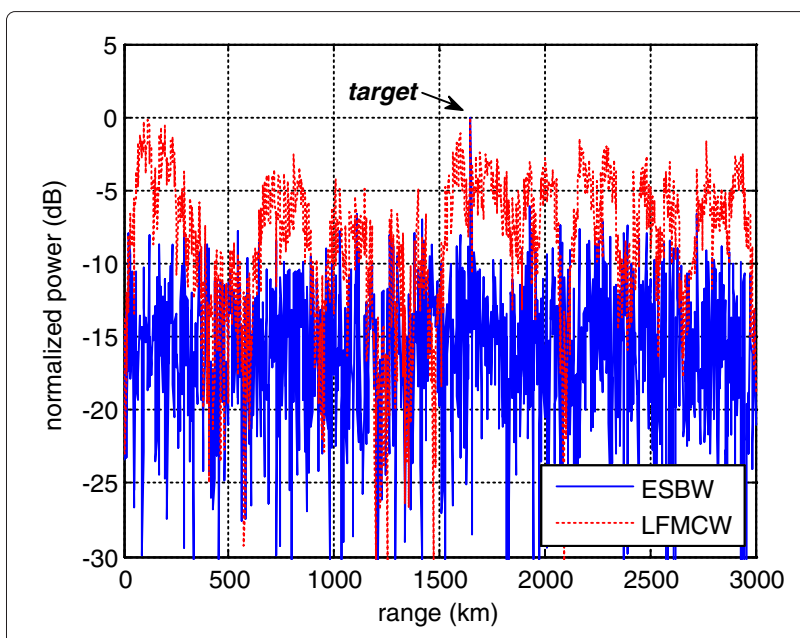

Figure 9 Power along range dimension at target velocity cell, normalized by the amplitude of target range cell.

normalized with respect to the power of the velocity cell where the target exists. For LFMCW, there are two peaks at velocity $\pm 165 \mathrm{~m} / \mathrm{s}$ produced by interference 1 as a result of Doppler ambiguity. Many lower peaks exist in other velocity cells, owing to $i_{2}(t)$ and $i_{3}(t)$. However, there is only one peak corresponding to the target velocity for ESBW. The peaks of $i_{1}(t)$ are canceled and those of $i_{2}(t)$ and $i_{3}(t)$ are suppressed to $-10 \mathrm{~dB}$ around and below.

Simulation results demonstrate that OTH radar employing ESBW achieves significant SNR improvement compared to LFMCW and detects the target successfully in the presence of strong interference. Recollect that the target echo and interference 2 share the same incidence angle $\theta_{t}=\theta_{2}$, and the steering vector of interference 3 is

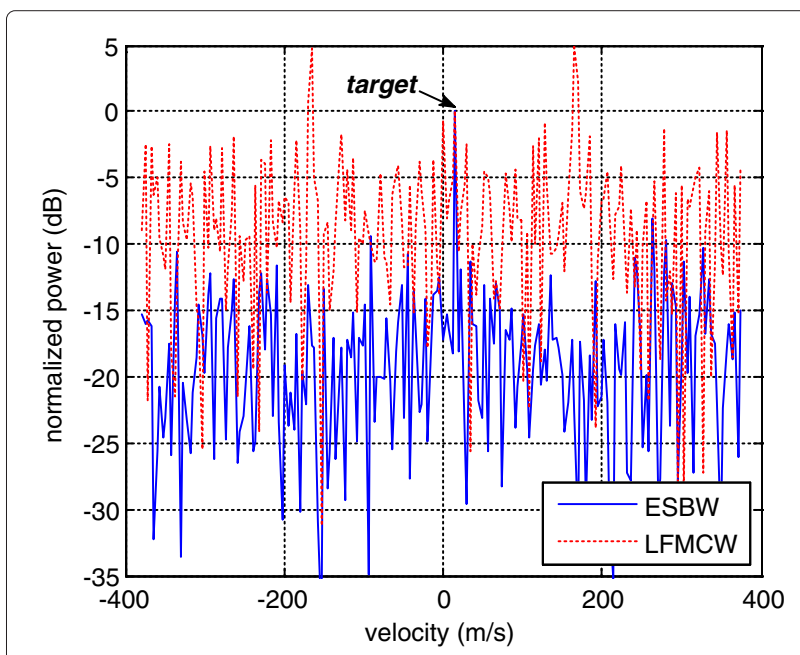

Figure 10 Power along velocity dimension at target range cell, normalized by the amplitude of target velocity cell. 
randomly produced. It illustrates that ESBW can suppress the interference from the mainlobe and colored noise without a clear incidence angle.

\subsection{Parameter analysis}

In the proposed ESBW strategy, there are two user parameters: monitoring time $T_{s}$ and similarity degree $\varepsilon$. Their effects on ESBW performance are important and worth investigating. In the following, detection SNR of ESBW strategy is simulated for various evaluations of $T_{s}$ and $\varepsilon$, with radar and environmental parameters set the same as previous subsections. Figure 11 depicts the statistical results by 2000 Monte Carlo simulations. It is worth noting that the detection SNR is about $17 \mathrm{~dB}$ when all three types of interference are absent in the environment (only AWGN), and $\varepsilon=0$ denotes LFMCW.

Firstly, observe the dependence of SNR on monitoring time $T_{s}$. It can be seen in Figure 11, SNR rises with $T_{s}$ mostly, for $\varepsilon$ in $0.005 \sim 0.5$. It is plain to see the reason that increasing monitoring time $T_{s}$ is good for EBSW design, since the environmental characteristic is better estimated. However, there is a reasonable value for $T_{s}$. Three lines behave similarly for $T_{s}=0.02$, 0.04 , and 0.1. Favorable SNR $(>15.5 \mathrm{~dB})$ is achieved for $T_{s}$ in $0.02 \sim 0.1$ and $\varepsilon$ in $0.05 \sim 0.5$. It reveals that $T_{s}$ greater than $0.02 \mathrm{~s}$ does not bring noticeable effect. The monitored data sampled in $0.02 \mathrm{~s}$ gives enough information of environmental noise to support favorable result for ESBW design. It is unlikely to obtain significant SNR improvement by increasing $T_{S}$ once $T_{s}$ exceeds $0.02 \mathrm{~s}$.

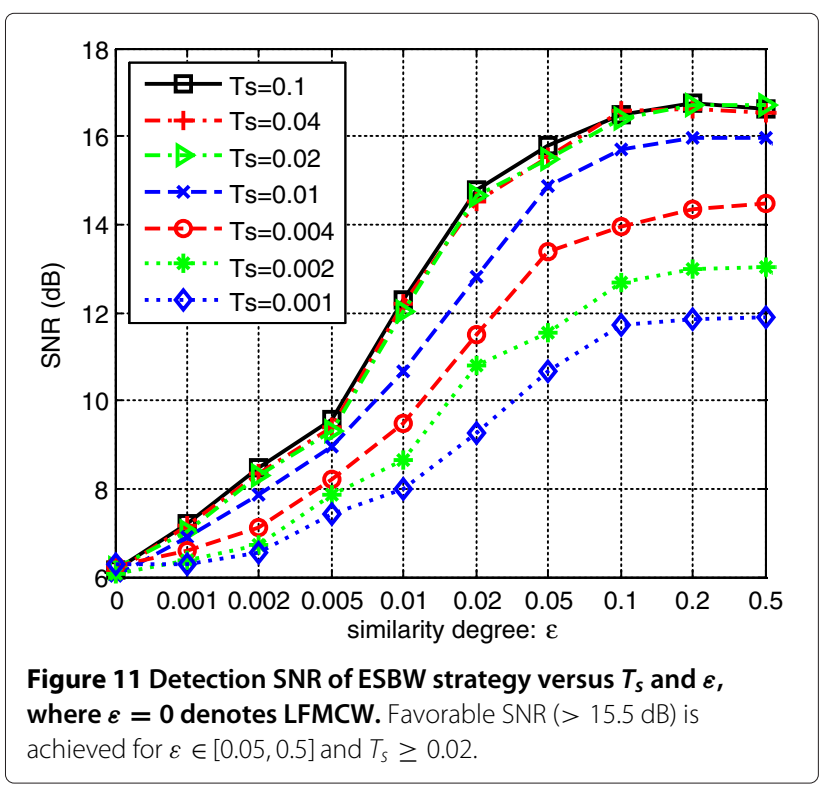

Secondly, observe the dependence of SNR on similarity degree $\varepsilon$. It can be seen that mostly, SNR grows along with $\varepsilon$, since greater $\varepsilon$ broadens the range of waveform design. However, SNR almost remains unchanged as $\varepsilon$ grows after 0.1 , unlike the theoretic SNR gain increasing with respect to $\varepsilon$ monotonically in (31). Figure 12 shows the power spectrum of ESBW for $\varepsilon=0.02,0.1$, and $0.5, T_{s}=0.04$. In Figure 12, as $\varepsilon$ grows, the power spectra of ESBW matches the PSD of environment noise better, with deeper and wider nulls and more detailed amplitude adjustment. However, greater $\varepsilon$ means that ESBW differs more from the desired waveform. Accordingly, for growing $\varepsilon$, the ACF bears higher sidelobe levels which would degrade the output SNR of moving MF, as shown in Figure 13. Besides, the AWGN cannot be suppressed, though the estimated covariance of AWGN is not an identity matrix and seems to allow the possibility.

At first glance, Figure 11 shows that SNR improvement can be enhanced by increasing $T_{s}$ or $\varepsilon$ alternatively. However, there are compromises in both $\varepsilon$ and $T_{s}$ evaluation, for the sake of reasonable ACF and conventional operation time for $\mathrm{OTH}$ radar. Herein, the reasonable advice is to locate $T_{s}$ in $0.02 \sim 0.1 \mathrm{~s}$ and $\varepsilon$ in $0.05 \sim 0.2$, to achieve desirable SNR improvement and ACF, as well as least reduction of radar operation time. It is worth noting that the reasonable evaluations of $T_{s}$ and $\varepsilon$ may vary for different environmental noise and radar system parameters (pulse length and bandwidth, etc.). Basically, it is concluded that reasonable monitoring time could be short for steady interference, and great similarity degree $\varepsilon$ is needed for severe occupation by interference. Therefore, OTH radar employing ESBW strategy prefers to take channels occupied by steady interference with narrow bandwidth.

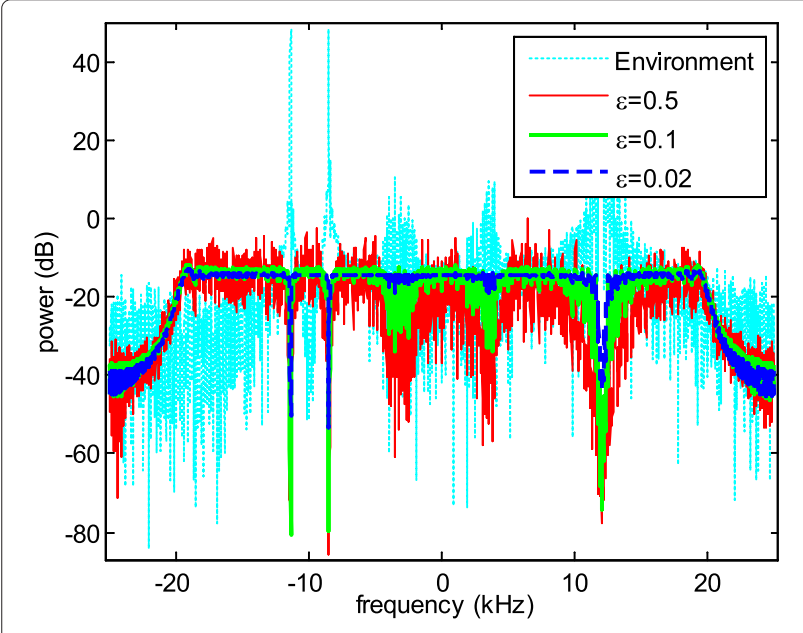

Figure 12 Power spectra of ESBW, for $\varepsilon=0.5,0.1,0.02$, $B=40 \mathrm{kHz}, T_{s}=0.04$. 


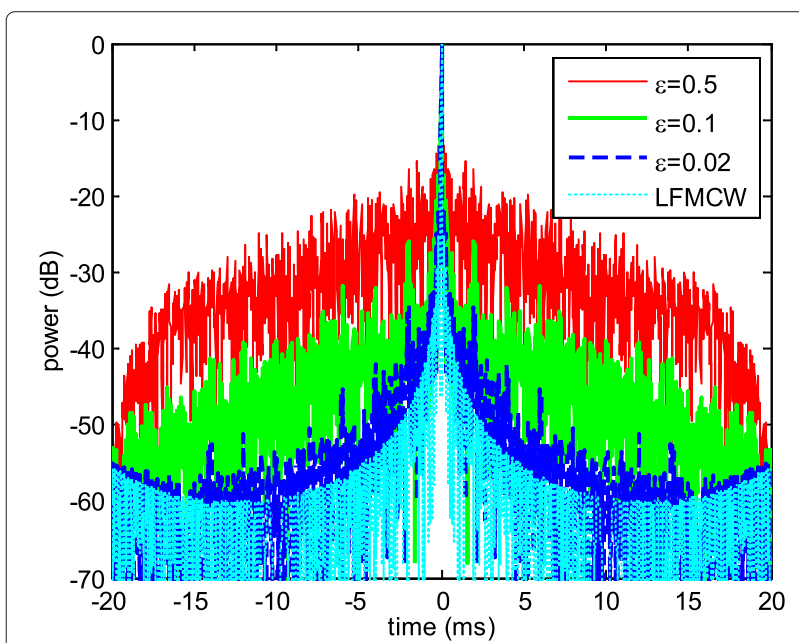

Figure 13 ACFs of LFMCW and ESBW, for $\varepsilon=0.5,0.1,0.02$, $T_{s}=0.04$.

\section{Conclusion}

This paper considers the problem of OTH radar employing wideband signal for ship detection in wanted bands occupied by cochannel interference. A cognitive waveform design method called 'ESBW strategy' is proposed, with several advantages or points as follows. (a) Waveform design is adaptively based on environmental sensing in real time, so ESBW strategy performs a cognitive cycle and works in the presence of non-stationary interference. (b) ESBW strategy is capable of detecting targets and suppressing interference from mainbeam direction or extended sources, by traditional receive beamforming, pulse compression (matched filter), and Doppler processing, in no need of extra interference cancelation algorithms. (c) Transmit waveform is optimized to maximize the SINR at the output of matched filter, under similarity constraint for desired range resolution and reasonable sidelobe levels indirectly. (d) The optimal solution to this constrained optimization is derived in closed form, so that the waveform design algorithm's convergence and efficiency is guaranteed. Numerical examples demonstrate that ESBW strategy suppresses interference successfully, achieves significant SINR improvements, and maintains the same range resolution essentially, compared to the widely used linear frequency modulated waveform.

\section{Appendix}

\section{Waveform Design for Maximizing SINR}

Herein, we prove that waveform design based on the criteria of maximizing the output SINR of array signal processing can be formulated as optimization problem in (8).

Consider an OTH radar with one omnidirectional transmitting antenna and uniform linear receive array consisting of $K$ antennas with $d$ distance spacing between adjacent antennas. During radar operation, the transmitter modulates discrete waveform $\boldsymbol{s}$ to carrier frequency $f_{c}$ in HF band and illuminates the area of interest. Assume that radar signal is scattered by a target, and the echo arrives at receive array with incidence angle $\theta$. Receive array modulates target echo and cochannel interference to base band, takes samples in discrete time. The signal model of radar received data is given by

$$
\boldsymbol{r}\left(t_{n}\right)=\boldsymbol{s}_{t}\left(t_{n}\right)+\boldsymbol{r}_{\boldsymbol{i}}\left(t_{n}\right),
$$

where $t_{n}$ denotes the sample time, $s_{t}\left(t_{n}\right)$ denotes the received target echo, and $\boldsymbol{r}_{\boldsymbol{i}}\left(t_{n}\right)$ denotes environmental noise, including interference and additive noise.

Assume that the target location (slant range) and radial velocity are $R_{t}$ and $v_{t}$, respectively. Then, the received echo is given by

$$
\begin{aligned}
\boldsymbol{s}_{t}\left(t_{n}\right)= & \boldsymbol{a}^{T}(\theta) \cdot \alpha_{t} \sum_{p=0}^{P-1} \operatorname{rect}\left[\left(t_{n}-\tau-p T\right) / T\right] \\
& \cdot \boldsymbol{s}\left(t_{n}-\tau-p T\right) e^{j 2 \pi f_{d} \cdot p T},
\end{aligned}
$$

where $\alpha_{t}$ is a complex amplitude representing the effects of reflection coefficient and path loss, rect( $(\cdot)$ denotes rectangular window, $\operatorname{rect}(t)=1$ for $t \in(0,1)$, and otherwise $\operatorname{rect}(t)=0, T$ denotes the pulse length (i.e. repetition period for continuous waveform), and $P$ denotes the number of periods in CIT. The time delay, Doppler shift, and steering vector are

$$
\begin{aligned}
\tau & =2 R_{t} / c, \\
f_{d} & =2 v_{t} f_{c} / c, \\
\boldsymbol{a}(\theta) & =\left[e^{j 2 \pi f_{c} / c \cdot d \sin \theta \cdot 0}, \ldots, e^{j 2 \pi f_{c} / c \cdot d \sin \theta \cdot(K-1)}\right],
\end{aligned}
$$

respectively.

Environmental noise consists of interference and additive noise, whose signal model is given by

$$
\boldsymbol{r}_{i}\left(t_{n}\right)=\sum_{q=1}^{Q} \alpha_{q} \boldsymbol{b}_{q}^{T} \boldsymbol{i}_{q}\left(t_{n}\right)+\boldsymbol{n}\left(t_{n}\right)
$$

where $Q$ denotes the number of interference, $\boldsymbol{i}_{q}\left(t_{n}\right)$ denotes the $q$ th interference (colored noise as well), and row vector $\boldsymbol{b}_{q}$ contains the amplitudes of interference $\boldsymbol{i}_{q}\left(t_{n}\right)$ at $K$ antennas. Suppose that additive noise is spatial and temporal white Gaussian noise with zero mean, so $\boldsymbol{n}\left(t_{n}\right)$ is a $K \times P M$ matrix with elements identically independently distributed.

In signal processing, a $K$-dimensional row vector $\boldsymbol{w}$ is employed for receive beamforming, which yields

$$
\boldsymbol{x}\left(t_{n}\right)=\boldsymbol{w} \cdot \boldsymbol{r}\left(t_{n}\right) .
$$


Then, $s$ is employed for moving MF and FFT for Doppler processing. Assume that the target ought to exist in delayDoppler cell $\left(\tau^{\prime}, f_{d}^{\prime}\right)$, where the output amplitude is given by

$$
y\left(\tau^{\prime}, f_{d}^{\prime}\right)=x\left(t_{n}\right) \cdot s_{\mathrm{Ft}}^{H}\left(t_{n}, \tau^{\prime}, f_{d}^{\prime}\right),
$$

where

$$
\begin{aligned}
& \boldsymbol{s}_{\mathrm{Ft}}\left(t_{n}, \tau^{\prime}, f_{d}^{\prime}\right) \\
& =\left[\boldsymbol{s}\left(t_{n}-\tau^{\prime}\right) e^{j 2 \pi f_{d}^{\prime} 0 T}, \cdots, \boldsymbol{s}\left(t_{n}-\tau^{\prime}\right) e^{j 2 \pi f_{d}^{\prime}(P-1) T}\right]
\end{aligned}
$$

is a $P M$-dimensional vector. Rewrite (45) as

$$
\begin{aligned}
y\left(\tau^{\prime}, f_{d}^{\prime}\right)= & \boldsymbol{w} \boldsymbol{a}^{T}(\theta) \cdot \alpha_{t} \sum_{p=0}^{P-1} e^{j 2 \pi\left(f_{d}-f_{d}^{\prime}\right) p T} \boldsymbol{s}\left(t_{n}-\tau\right) \boldsymbol{s}^{H}\left(t_{n}-\tau^{\prime}\right) \\
& +\boldsymbol{w} \boldsymbol{r}_{\boldsymbol{i}}\left(t_{n}\right) \cdot \boldsymbol{s}_{\mathrm{Ft}}^{H}\left(t_{n}, \tau^{\prime}, f_{d}^{\prime}\right) .
\end{aligned}
$$

The first item of the right-hand side of (47) represents the target echo component, whose energy is given by

$$
\begin{aligned}
P_{t}= & \left|\boldsymbol{w a} \boldsymbol{a}^{T}(\theta)\right|^{2} \cdot\left|\alpha_{t} \sum_{p=0}^{P-1} e^{j 2 \pi\left(f_{d}-f_{d}^{\prime}\right) p T}\right|^{2} \cdot \mid \boldsymbol{s}\left(t_{n}-\tau\right) \\
& \times\left.\boldsymbol{s}^{H}\left(t_{n}-\tau^{\prime}\right)\right|^{2} .
\end{aligned}
$$

The second item of the right-hand side of (46) represents the interference and noise component, whose energy is given by

$$
P_{\boldsymbol{i}}=\mathcal{E}\left(\left|\boldsymbol{w} \boldsymbol{r}_{\boldsymbol{i}}\left(t_{n}\right) \cdot \boldsymbol{s}_{\mathrm{Ft}}^{H}\left(t_{n}, \tau^{\prime}, f_{d}^{\prime}\right)\right|^{2}\right)
$$

where $\mathcal{E}(\cdot)$ denotes the expectation with respect to the random variables within the bracket. By denoting $I=$ $\boldsymbol{w} \boldsymbol{r}_{\boldsymbol{i}}\left(t_{n}\right)$, we arrive at

$$
P_{\boldsymbol{i}}=\boldsymbol{s}_{\mathrm{Ft}}\left(t_{n}, \tau^{\prime}, f_{d}^{\prime}\right) \cdot \mathcal{E}\left(\boldsymbol{I}^{H} \boldsymbol{I}\right) \cdot \boldsymbol{s}_{\mathrm{Ft}}^{H}\left(t_{n}, \tau^{\prime}, f_{d}^{\prime}\right) .
$$

Assume that each interference is wide-sense stationary (w.s.s.), and so $I$ is w.s.s. too, with covariance matrix

$$
\boldsymbol{R}_{P}=\mathcal{E}\left(\boldsymbol{I}^{H} \boldsymbol{I}\right)
$$

Hence, by (48) and (50), the SINR at delay-Doppler cell $\left(\tau^{\prime}, f_{d}^{\prime}\right)$ is calculated

$$
\begin{aligned}
\mathrm{SINR} & =P_{t} / P_{\boldsymbol{i}} \\
& =\frac{\left|\boldsymbol{w} \boldsymbol{a}^{T}(\theta)\right|^{2} \cdot\left|\alpha_{t} \sum_{p=0}^{P-1} e^{j 2 \pi\left(f_{d}-f_{d}^{\prime}\right) p T}\right|^{2} \cdot\left|\boldsymbol{s}\left(t_{n}-\tau\right) \boldsymbol{s}^{H}\left(t_{n}-\tau^{\prime}\right)\right|^{2}}{\boldsymbol{s}_{\mathrm{Ft}}\left(t_{n}, \tau^{\prime}, f_{d}^{\prime}\right) \cdot \boldsymbol{R}_{P} \cdot \boldsymbol{s}_{\mathrm{Ft}}^{H}\left(t_{n}, \tau^{\prime}, f_{d}^{\prime}\right)} .
\end{aligned}
$$

Considering that OTH radar employs large pulse length for improving transmitting energy and range unambiguity in ship detection, we suppose that interference covariance function concentrates most energy within interval time $T$ and spreads little out. Thus $\boldsymbol{R}_{P}$ in (51) is approximated as

$$
\boldsymbol{R}_{P}=\left[\begin{array}{cccc}
\boldsymbol{R}_{\boldsymbol{i}} & \mathbf{0}_{M} & \cdots & \mathbf{0}_{M} \\
\mathbf{0}_{M} & \boldsymbol{R}_{\boldsymbol{i}} & \cdots & \mathbf{0}_{M} \\
\vdots & \vdots & \ddots & \vdots \\
\mathbf{0}_{M} & \mathbf{0}_{M} & \cdots & \boldsymbol{R}_{\boldsymbol{i}}
\end{array}\right]
$$

where $\boldsymbol{R}_{\boldsymbol{i}}$ denotes the $M$-rank covariance matrix of $\boldsymbol{I}$, and $\mathbf{0}_{M}$ denotes an $M$-rank zero matrix. Plugging (46) and (53) in (52) yields

SINR

$$
=\frac{\left|\boldsymbol{w} \boldsymbol{a}^{T}(\theta)\right|^{2} \cdot\left|\alpha_{t} \sum_{p=0}^{P-1} e^{j 2 \pi\left(f_{d}-f_{d}^{\prime}\right) p T}\right|^{2} \cdot\left|\boldsymbol{s}\left(t_{n}-\tau\right) \boldsymbol{s}^{H}\left(t_{n}-\tau^{\prime}\right)\right|^{2}}{P \cdot \boldsymbol{s}\left(t_{n}-\tau^{\prime}\right) \boldsymbol{R}_{i} \boldsymbol{s}^{H}\left(t_{n}-\tau^{\prime}\right)} .
$$

Without loss of generality, assume $f_{d}=f_{d}^{\prime}, \tau=\tau^{\prime}=0$. Then, formula (54) is rewritten as

$$
\operatorname{SINR}=\frac{\left|\boldsymbol{w} \boldsymbol{a}^{T}(\theta)\right|^{2} \cdot P\left|\alpha_{t}\right|^{2} \cdot\left|\boldsymbol{s} \boldsymbol{s}^{H}\right|^{2}}{\boldsymbol{s} \boldsymbol{R}_{\boldsymbol{i}} \boldsymbol{s}^{H}} .
$$

Hereby, the problem of maximizing SINR in (55) by designing $s$ is equivalent to the following optimization problem

$$
\min _{\boldsymbol{s}} \boldsymbol{s}_{\boldsymbol{i}} \boldsymbol{s}^{H}, \quad \text { s.t. } \quad \boldsymbol{s} \boldsymbol{s}^{H}=1 .
$$

The definition of $\boldsymbol{R}_{\boldsymbol{i}}$ is as follows. Covariance function of $I$ is defined as

$$
R_{\boldsymbol{i}}(m)=\mathcal{E}\left\{I(l+m) \cdot I^{*}(l)\right\}, 0 \leq m \leq M-1 .
$$

where $I(l)$ denotes the $l$ th element of $I$. Then, covariance matrix $\boldsymbol{R}_{\boldsymbol{i}}$ is obtained by arranging $\boldsymbol{R}_{\boldsymbol{i}}(m)$ like (7). Obviously, $\boldsymbol{R}_{\boldsymbol{i}}$ is a Toeplitz matrix and $\boldsymbol{R}_{\boldsymbol{i}}=\boldsymbol{R}_{\boldsymbol{i}}^{H}$.

As two-order characteristic property of time-varying environment noise, $\boldsymbol{R}_{\boldsymbol{i}}$ is generally thought to be unknown. Herein for the optimization problem in (56), covariance matrix estimate $\widehat{\boldsymbol{R}}_{\boldsymbol{i}}$ is used instead of $\boldsymbol{R}_{\boldsymbol{i}}$. Based on environment data collected in step 'environment monitoring', covariance function estimate $\widehat{R}_{\boldsymbol{i}}(m)$ is obtained by (5) or (6), and then covariance matrix estimate $\widehat{\boldsymbol{R}}_{i}$ is achieved by (7) in step 'characteristic estimating'. By replacing $\widehat{\boldsymbol{R}}_{\boldsymbol{i}}$ with $\boldsymbol{R}_{\boldsymbol{i}}$ in (56), the optimization problem is formulated as (8).

Competing interests

The authors declare that they have no competing interests.

\section{Acknowledgements}

This work was supported by the National Nature Science Foundation of China under Grants 61032010 and 61102142 and the cooperation foundation between Nanjing Research Institute of Electronics Technology (NRIET) and University of Electronic Science and Technology of China (UESTC). 


\section{Author details}

${ }^{1}$ University of Electronic Science and Technology of China, Xiyuan Ave, West Hi-Tech Zone, 611731 Chengdu, China. ${ }^{2}$ Nanjing Research Institute of Electronics Technology, Guorui Road, Yuhua District, 210039 Nanjing, China.

Received: 16 May 2014 Accepted: 22 October 2014

Published: 28 October 2014

\section{References}

1. JM Headrick, MI Skolnik, Over-the-horizon radar in the HF band. Proc. IEEE 62(6), 664-673 (1974)

2. GA Fabrizio, High Frequency Over-the-Horizon Radar: Fundamental Principles, Signal Processing, and Practical Applications (McGraw-Hill, NY, 2013)

3. G Earl, B Ward, Frequency management support for remote sea-state sensing using the Jindalee skywave radar. IEEE J. Ocean. Eng. 11(2), 164-173 (1986)

4. V Bazin, JP Molinie, J Munoz, P Dorey, S Saillant, G Auffray, V Rannou, M Lesturgie, Nostradamus: an OTH radar. IEEE Aero. Electron. Syst. Mag. 21(10), 3-11 (2006)

5. KW Gurgel, Y Barbin, T Schlick, in OCEANS 2007-Europe. Radio frequency interferences suppression techniques in FMCW modulated HF radars (IEEE, Aberdeen, UK, 2007), pp. 1-4

6. BT Root, HF-over-the-horizon radar ship detection with short dwells using clutter cancellation. Radio Sci. 33(4), 1095-1111 (1998)

7. JM Headrick, SJ Anderson. 3rd, in Radar Handbook, ed. by MI Skolnik. HF over-the-horizon radar, vol. 20 (McGraw-Hill, NY, 2008)

8. AL Saverino, A Capria, F Berizzi, ED Mese, in 2010 2nd International Workshop on Cognitive Information Processing (CIP). Cognitive adaptive waveform technique for HF skywave radar (IEEE, Elba, Italy, 2010), pp. 247-252

9. GA Fabrizio, AB Gershman, MD Turley, in Proceedings of the International Radar Conference. Non-stationary interference cancellation in HF surface wave radar (IEEE, Adelaide, Australia, 2003), pp. 672-677

10. GA Fabrizio, YI Abramovich, SJ Anderson, DA Gray, MD Turley, Adaptive cancellation of nonstationary interference in HF antenna arrays. IEE Proc. Radar Sonar Navigation 145(1), 19-24 (1998)

11. X Luo, LMH Ulander, J Askne, G Smith, PO Frolind, RFI suppression in ultra-wideband SAR systems using LFM filters in frequency domain. Electron. Lett. 37(4), 241-243 (2001)

12. Z Hao, W Biyang, W Shicai, L Yuyang, Radio frequency interferences suppression in HF radars. Electron. Lett. 39(12), 925-927 (2003)

13. H Zhou, B Wen, $\mathrm{S}$ Wu, Dense radio frequency interferences suppression in HF radars. IEEE Signal Process. Lett. 12(5), 361-364 (2005)

14. X Guo, H Sun, TS Yeo, Interference cancellation for high-frequency surface wave radar. IEEE Trans. Geosci. Rem. Sens. 46(7), 1879-1891 (2008)

15. MJ Lindenfeld, Sparse frequency transmit and receive waveform design. IEEE Trans. Aero. Electron. Syst. 40(3), 851-861 (2004)

16. H He, P Stoica, J Li, in 2010 2nd International Workshop on Cognitive Information Processing (CIP). Waveform design with stopband and correlation constraints for cognitive radar (IEEE, Elba, Italy, 2010), pp. 344-349

17. LK Patton, CA Bryant, B Himed, in IEEE Radar Conference (RADAR). Radar-centric design of waveforms with disjoint spectral support (IEEE, Atlanta, USA, 2012), pp. 269-274

18. S Haykin, Cognitive radar: a way of the future. IEEE Signal Process. Mag. 23(1), 30-40 (2006)

19. K Lu, XY Chen, in IEEE CIE International Conference on Radar (Radar). Cognitive over-the-horizon radar (IEEE, Chengdu, China, 2011), pp. 993-996

20. LK Patton, BD Rigling, Phase retrieval for radar waveform optimization. IEEE Trans. Aero. Electron. Syst. 48(4), 3287-3302 (2012)

21. LK Patton, BD Rigling, in IEEE Radar Conference (Radar). Optimal signal/filter design for multi-target detection in colored noise (IEEE, Kansas, USA, 2011), pp. 276-281

22. LK Patton, SW Frost, BD Rigling, Efficient design of radar waveforms for optimised detection in coloured noise. IET Radar Sonar Navigation 6(1), 21-29 (2012)

23. S Sussman, Least-square synthesis of radar ambiguity functions. IEEE Trans. Inf. Theory 8(3), 246-254 (1962)
24. A De Maio, S De Nicola, H Yongwei, L Zhi-Quan, Z Shuzhong, Design of phase codes for radar performance optimization with a similarity constraint. IEEE Trans. Signal Process. 57(2), 610-621 (2009)

25. G Cui, H Li, H Rangaswamy, MIMO radar waveform design with constant modulus and similarity constraints. IEEE Trans. Signal Process. 62(2), 343-353 (2014)

26. J Li, P Stoica, Z Wang, Doubly constrained robust capon beamformer. IEEE Trans. Signal Process. 52(9), 2407-2423 (2004)

27. J Li, JR Guerci, L Xu, Signal waveform's optimal under restriction design for active sensing. IEEE Signal Process. Lett. 13(9), 565-568 (2006)

28. X Guo, JL Ni, GS Liu, Architecture and signal processing of sky wave over-the-horizon radar. Radio Sci. 38(5), 13/1-13/14 (2003)

29. TF Quan, JW Li, CJ Yu, H Wang, ZL Ma, An approach and experiment of suppressing burst interference in high-frequency radar. Acta Electron. Sinica 27(12), 23-25 (1999)

30. M Turley, in Proceedings of the International Radar Conference. Impulse noise rejection in $\mathrm{HF}$ radar using a linear prediction technique (IEEE, Adelaide, Australia, 2003), pp. 358-362

31. S Kay, Fundamentals of Statistical Signal Processing: Estimation Theory (Prentice-Hall, Inc, Upper Saddle River, 1993)

doi:10.1186/1687-6180-2014-159

Cite this article as: Luo et al.: Wideband signal design for over-the-horizon radar in cochannel interference. EURASIP Journal on Advances in Signal Processing 2014 2014:159.

\section{Submit your manuscript to a SpringerOpen ${ }^{\odot}$ journal and benefit from:}

- Convenient online submission

Rigorous peer review

- Immediate publication on acceptance

- Open access: articles freely available online

- High visibility within the field

- Retaining the copyright to your article

Submit your next manuscript at $\boldsymbol{\nabla}$ springeropen.com 